\title{
A case of non-occlusive mesenteric ischemia and hepatic portal venous gas: not everyone knows that...
}

\author{
Luisa Siciliani · Laura Riccardi • Angela Favuzzi • \\ Maurizio Pompili · GianLudovico Rapaccini
}

Received: 20 February 2011/Accepted: 14 March 2011/Published online: 31 March 2011

(C) SIMI 2011

Non-occlusive mesenteric ischemia (NOMI) includes all forms of mesenteric ischemia without occlusion of the mesenteric arteries [1]. It is mostly seen in patients who are undergoing dialysis, with an estimated incidence rate up to $1.9 \%$ per patient/year. In these patients, predisposing conditions such as peripheral vascular disease, atrial dysrhythmias, congestive heart failure, obstructive pulmonary disease, and insulin-dependent diabetes mellitus are precipitated by ultrafiltration with a resulting splanchnic hypoperfusion and gangrene of the bowel wall [2].

We present a case of an 87-year-old man who developed NOMI with portomesenteric venous gas and pneumomatosis intestinalis during his first weeks of haemodialysis.

The patient was referred to our Department of Ultrasound for diffuse abdominal pain that appeared after a dialysis session. He had a history of chronic renal failure (uremic phase, K/DOQI stage V), chronic atrial fibrillation, obstructive pulmonary disease, and heart failure (NYHA class 14IV), systemic hypertension, diabetes mellitus type II, normochromic normocytic anemia, peripheral arterial obstructive disease (Leriche-Fontaine stage IV) with multiple aneurysms (aortic, left iliac-femoral, hypogastric, right and left popliteal) treated with placement of prostheses and by-pass grafts.

He was admitted because of worsening dyspnea, and the appearance of impressive edema of the limbs. During hospitalization, medical therapy failed to improve his

Electronic supplementary material The online version of this article (doi:10.1007/s11739-011-0569-8) contains supplementary material, which is available to authorized users.

L. Siciliani $(\bowtie) \cdot$ L. Riccardi · A. Favuzzi · M. Pompili ·

G. Rapaccini

Rome, Italy

e-mail: luisa.siciliani@libero.it condition. Despite the maintenance of diuresis, renal failure caused hyperkalemia and a severe metabolic acidosis with an elevated level of uremic acid. Pulmonary edema persisted, and therefore it was decided to commence hemodialysis.

After 6 days of dialysis, the patient developed a swelling of the right thigh. A computed tomography (CT scan) with contrast was performed that showed a hematoma larger than $7 \mathrm{~cm}$, probably due to treatment with anticoagulants. The first hypotensive episode of short duration occurred, and was successfully treated with plasma expanders. At this time, the patient did not complain of abdominal pain.

Two days later, the patient underwent a new dialysis session, and was then sent for our evaluation. The blood pressure was $90 / 60 \mathrm{mmHg}$, and the pulse rate 90 beats $/ \mathrm{min}$. The patient reported constipation, but denied vomiting. He neither has fever nor dyspnea. The abdomen was distended and tympanitic, without guarding, and with hypoactive bowel sounds. The extremities were cold and the skin was pallid showing signs of peripheral vasoconstriction.

Laboratory measurements revealed: haemoglobin $6.7 \mathrm{~g} /$ $\mathrm{dl}$, hematocrit $21.1 \%$, white blood cell (WBC) count $8.47 \times 10^{9} 1^{-1}$ with $87.3 \%$ neutrophils.

Biochemistry showed serum creatinine $3.9 \mathrm{mg} / \mathrm{dl}$, normal lactic dehydrogenase (361 UI/l) with high level of creatine kinase (291 UI/l). INR was 1.68, APTT $43.60 \mathrm{~s}$. Venous blood gas analysis was: $\mathrm{pH}$ 7.356, $\mathrm{pCO}_{2}$ $38 \mathrm{mmHg}, \mathrm{HCO}_{3} 20.9 \mathrm{mEq} / \mathrm{l}$ and standard base excess $3.9 \mathrm{mEq} / \mathrm{l}$. Peripheral oxygen saturation was undetectable.

Liver ultrasonography revealed patchy small hyperechoic areas, due to gas in the peripheral portal vein branches (Fig. 1a, b, arrowheads). Second harmonic ultrasound identified in the lumen of the right portal vein and in the main portal trunk small hyperechoic foci due to gas 

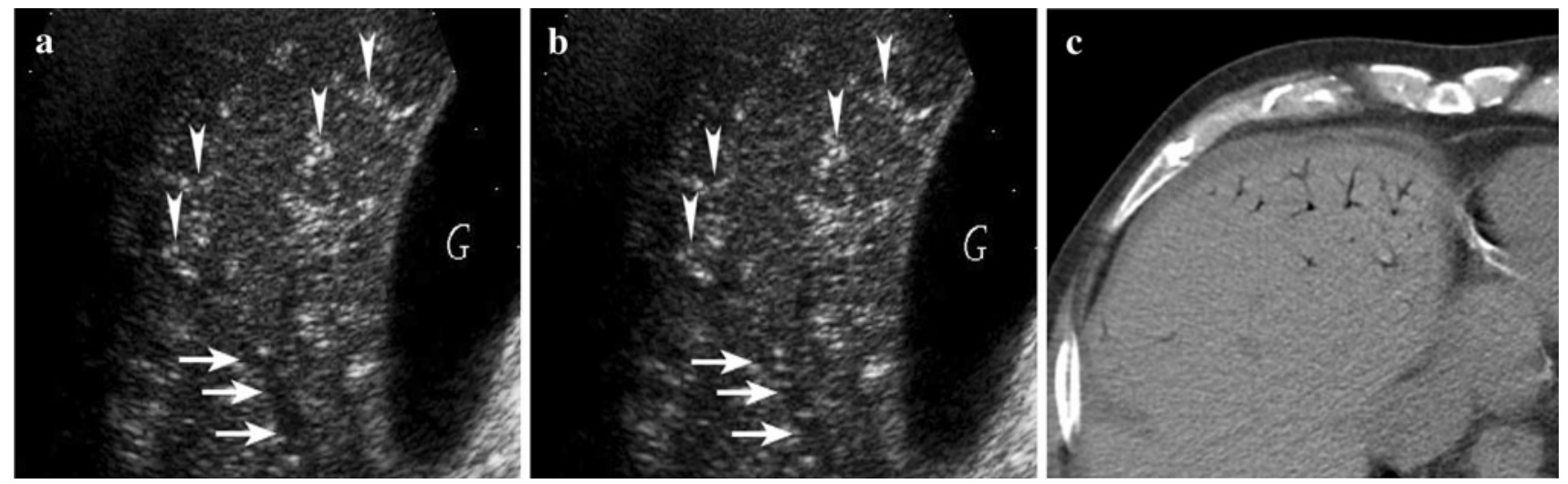

Fig. 1 a-b Ultrasonographic details of two consecutive frames extract from same clip. Right portal branch is evaluated by a second harmonic probe: bubbles not yet visible in frame (a) appear in the vessel of frame (b) (arrows). Aeroportia is present in $>50 \%$ of the

bubbles resembling a 'string of pearls' moving in the direction of blood flow (Fig. 1a, b, arrows). Doppler evaluation of the right portal vein revealed amplification of signals due to gas motions; typical wide spikes were sampled, superimposed on a normal portal trace. Based upon these findings, bowel ischemia was suspected.

An abdominal CT scan was performed a few hours later. It showed linear tubular hypoattenuating areas in the periphery of both lobes of the liver, within $2 \mathrm{~cm}$ of the capsule, band-like pneumomatosis intestinalis (PI), airfluid levels of jejunal loops, tubular areas of low attenuation in the mesentery (consistent with air in the mesenteric vein branches), gas in the superior mesenteric vein and peritoneal effusion.

Despite aggressive medical treatment, the patient died $48 \mathrm{~h}$ after the onset of the shock.

NOMI accounts for $20-30 \%$ of all case of acute mesenteric ischemia [1], and is considered a rare pathology among the general population with an incidence of $0.09-0.2 \%$ per patient/year [2]. Clinical signs of NOMI and laboratory data are not specific.

Despite technical evolution of non-invasive methods, angiography is still a gold standard in diagnosing peripheral splanchnic vasospasm induced by hypotension [1]. Unfortunately, the poor hemodynamic status of the patient precluded performing angiography or laparotomy [2], and NOMI remained a likely but unproven diagnosis.

This condition should be thought of and looked for in every hemodialytic patient with severe abdominal pain, particularly if the pain is associated with arterial hypotension. The characteristic risk factor constellation and the triggering factors predisposing to NOMI should be identified.

Our patient was elderly, affected by multiple aneurysmal dilatations, heart failure, chronic obstructive pulmonary disease, arterial hypertension, diabetes mellitus, and lobe (arrowheads). c ACT examination was performed a few hours later, and confirmed the US diagnosis of aeroportia with extensive bowel necrosis ( $G$ gallbladder)

peripheral vascular disease, all of which are considered relevant pathological conditions able to alter circulation.

Postmortem, the contrast-enhanced CT scan performed for the diagnosis of the hematoma ( 3 days prior to the patient's death) was reviewed to exclude an occlusive bowel ischemia and to confirm NOMI. Diffuse atherosclerosis-induced stenoses were found in the hepatic, splenic, left gastric arteries, in the abdominal aorta, and in the superior and inferior mesenteric arteries. Atheromatosis of inferior mesenteric artery was severe, and the vessel was partly perfused by collaterals. Pneumomatosis and portal venous gas were not detected on this examination.

These data strongly suggest that the development of the hematoma and volume depletion induced by a fast and excessive ultrafiltration during dialysis sessions favored the occurrence of bowel necrosis in a patient with an advanced condition of mesenteric hypoperfusion.

Dialysis was certainly inevitable in this patient, and the risk of NOMI had to be run, but NOMI could possibly have been avoided by a less 'aggressive' treatment (i.e. less frequent and shorter sessions of dialysis in this patient whose diuresis was maintained, or with the use of plasma expanders and positive inotropic factors after each session).

HPVG is frequent in NOMI but rarely described.

Today there is not a general consensus in considering portal venous gas as certain sign of fatal outcome. Mortality rises when HPVG is combined with PI, and severe abdominal pain or paucity of abdominal findings. The review of the literature reveals that neither portomesenteric venous gas nor PI are pathognomic of bowel infarction $[2,3]$. HPVG occurs under various conditions including endoscopy, organ transplant (liver, kidney, and bone marrow), digestive tract distention, gastric ulcer, ulcerative colitis, Crohn's disease, severe enteritis, acute necrotizing pancreatitis, graft-versus-host reaction, and pulmonary diseases. 
Kinoshita et al. report on a cumulative review of the literature. They find 182 cases of HPVG: most of these patients (79, that was $43 \%$, the major percentage) had bowel necrosis with a mortality of $75 \%$; one patient had fulminant hepatitis and the mortality was $100 \%$; the remnant had other pathological conditions with a low mortality $(<30 \%)$; the overall mortality described was $39 \%[4,5]$.

In our opinion, HPVG must be always considered as a sign of poor prognosis with a high mortality, until evidence of bowel ischemia and life-threatening clinical conditions have been ruled out. Its diagnosis is difficult, and it is a rare condition. Although case reports with a low mortality have been described, they should not be misperceived as common conditions.

In conclusion, NOMI is a rare entity that often occurs in hemodialysis patients. It is of paramount importance to identify and prevent the pathogenetic factors increasing the risk of NOMI because this represents the only chance to improve the rate of survival, since its therapy is difficult to manage. CT scanning in association with ultrasonography represents useful tools to make a prompt diagnosis of HPVG and PI that must be always considered as sign of poor prognosis.
Conflict of interest None.

\section{References}

1. Trompeter M, Brazda T, Remy CT, Vestring T, Reimer P (2002) Non-occlusive mesenteric ischemia: etiology, diagnosis, and interventional therapy. Eur Radiol 12(5):1179-1187

2. Picazo M, Cuxart M, Sans R, Sardá C, Expósito E (2008) Mesenteric ischemia in hemodialysis patients. Nefrologia 28(2): 198-202

3. Lai WH, Hwang TL, Chen HW (2008) Portomesenteric venous gas in acute bowel ischemia: report of a case. Surg Today 38(7):656-660

4. Wiesner W, Mortelé KJ, Glickman JN, Ji H, Ros PR (2001) Pneumatosis intestinalis and portomesenteric venous gas in intestinal ischemia: correlation of CT findings with severity of ischemia and clinical outcome. Am J Roentgenol 177(6):13191323

5. Kinoshita H, Shinozaki M, Tanimura H et al (2001) Clinical features and management of hepatic portal venous gas: four case reports and cumulative review of the literature. Arch Surg 136(12):1410-1414 